\title{
Letter to the Editor: Prevalence of Equinus in Patients Diagnosed with Plantar Fasciitis
}

\author{
by Robert D. Phillips, DPM ${ }^{1}$
}

The Foot and Ankle Online Journal 2 (4): 5

Accepted: March, 2009

Published: April, 2009

This is an Open Access article distributed under the terms of the Creative Commons Attribution License. It permits unrestricted use, distribution, and reproduction in any medium, provided the original work is properly cited. @The Foot and Ankle Online Journal (www.faoj.org)

\section{To the editor:}

I read with interest the article by Wenzel, et al., on the relationship between equinus and plantar fasciitis. I feel the authors should be commended for the work they have done in trying to better define the pathomechanics of this syndrome that is so wide spread today. In regards to their findings, I would like to make a few observations and comments.

The authors acknowledge that there is a variation in the literature in accepted dorsiflexion values that are necessary for normal gait, and settle on using the most commonly cited value of $10^{\circ}$ as the cut-off point. This magical demarcation point often puzzled me until I began writing chapter 3 for the book Principles and Practices of Podiatric Medicine ${ }^{1}$ in which I looked at the passive and active moments created around each joint of the lower extremity during gait. In doing some calculations, I found that for a person of average weight and height and foot length, when the ankle joint dorsiflexed $10^{\circ}$, the center of body mass would pass anterior to the metatarsophalangeal joints. This then made it clear that when the center of body mass is posterior to the metatarsophalangeal joints, the weight of the body places a plantarflexion moment around the metatarsophalangeal joints, and when the center of body mass is anterior to the metatarsophalangeal joints, the body weight places a dorsiflexion moment around the metatarsophalangeal joints.

\footnotetext{
Address correspondence to: Robert D. Phillips, D.P.M.

${ }^{1}$ Orlando Veterans Affairs Medical Center, Orlando, FL 32803
}

Pronation dysfunction of the midfoot and rearfoot joints could then be explained by the Achilles tendon trying to lift the heel before the center of body mass passed anterior to the metatarsophalangeal joints.

In light of this argument, it makes sense that there are a number of parameters, which if altered, could make it possible for a person to need more or less than $10^{\circ}$ of ankle joint dorsiflexion in order to walk with a normal gait. For example, anything that moves the center of body mass upward would decrease the need for $10^{\circ}$ of ankle joint dorsiflexion and anything that moves the center of body mass downward would increase the need for $10^{\circ}$ of ankle joint dorsiflexion in gait. For example, just holding one's hands on top of the head when walking moves the center of body mass upward. In comparison, carrying two heavy weights in one's hands moves the center of body mass downward. A person carrying a heavy load on the head would mean that less than $10^{\circ}$ of ankle joint dorsiflexion may be needed and a person carrying a heavy suitcase would mean that more than $10^{\circ}$ of ankle joint dorsiflexion. Body morphology also may place the center of body mass naturally higher or lower. A person who has a robust upper body and a slender lower body phenotype would need less than $10^{\circ}$ of dorsiflexion, whereas a person who had an extreme amount of edema in the legs, or had a phenotype that concentrated weight well below the waist would need more than $10^{\circ}$ of dorsiflexion. 
This argument could easily explain the authors' finding that people with greater BMI, especially females, would be more affected by decreased ankle joint dorsiflexion. Also it would explain why very young children, who have a much shorter leg in proportion to their height need much more than $10^{\circ}$ of dorsiflexion.

Other parameters that would move the center of body mass more anterior or more posterior could mean that less or more than $10^{\circ}$ of ankle joint dorsiflexion would be needed. If the body weight is distributed more posteriorly, then more than $10^{\circ}$ of dorsiflexion will be needed, whereas carrying the center of body mass more anteriorly would decrease the need for $10^{\circ}$ of ankle joint dorsiflexion. The length of the foot is also an important parameter. People with shorter feet relative to the body height should need less than $10^{\circ}$ of ankle joint dorsiflexion whereas those with longer foot length relative to their body height should need more than $10^{\circ}$ of ankle joint dorsiflexion.

The tension in the Achilles tendon can also be broken down into two components, the active tension component and the passive tension component. The active tension component is proportional to the contractile force exerted by the muscle fibers, however the passive component is set by a fixed length tension curve for that particular tendon. I attempted many years ago to determine in-vivo the passive length tension curve for the Achilles tendon in individuals, but found that the problem was greater than the then-current capabilities of our equipment. However, I do believe that this may be doable in the future with improvements and refinements in technology. This has been brought to the fore by recent articles (quoted by the authors) showing a correlation between tightness in the Achilles tendon and the force under the forefoot in diabetics. Indeed, in diabetics, glycosylation of the collagen fibers, moves the passive length tension curve to the left, which means that it takes more force against the forefoot to stretch the Achilles to a point of the ankle being $10^{\circ}$ dorsiflexed. As a result, procedures that lengthen the Achille Tendon are coming in vogue for decreasing the risk of forefoot ulcerations in diabetics.
The authors did report the results of ankle joint dorsiflexion with the knee extended and knee flexed, and it is inferred in the article that the equinus group included those who had less than $10^{\circ}$ of motion with the knee extended, no matter what the range of motion was with the knee flexed. Indeed, it may be critical to divide these into two very different types of equinus conditions. This is because if a person has a marked difference between the ankle joint dorsiflexion with the knee flexed and extended, it could have a marked difference in the percent contribution of the passive tensions to the total tension in the Achilles tendon before the heel comes off the ground. Unfortunately, the literature has not well addressed the situation of isolated gastrocnemius tightness to the distribution of forces in the Achilles tendon, verses when both the gastrocnemius and soleus are tight. Theoretically - because of the rotation of the Achilles tendon, such that the soleus inserts more to the medial side of the os calcis and the gastrocnemius inserts more to the lateral side of the os calcis - if only the gastrocnemius is tight, then as the ankle joint dorsiflexes there would result an overload of stress more in the lateral side of the insertion of the Achilles, which would tend to have more of a pronatory moment around the subtalar joint axis. On the other hand if the ankle joint dorsiflexion changes little between the knee being flexed and being extended, it would have less of an eversion torque on the heel, but a greater dorsiflexion moment around the transverse tarsal joint, dorsiflexing the naviculocuboid complex against the talocalcaneal complex. This type of tight Achilles may show less pronation of the rearfoot, especially if it is an adult acquired tightness, yet have a marked increase in the tension of the plantar fascia as it resists dorsiflexion moments around the midtarsal joint. This argument would reinforce the authors' finding that those with both gastrocnemius and soleus tightness were more likely to have plantar fasciitis than those only with gastrocnemius equinus, without having as much pes planus effect.

In light of these, plus other variables that I have not addressed, I believe it is important that one not put too much faith in the lack of findings of no statistical relationship between the presence of equinus and the presence of plantar fasciitis. 
Certainly, the tables show a trend in that direction, but because of the wide standard deviation, the rejection of the null hypothesis at the $95 \%$ confidence level cannot be made. The purpose of this letter is to begin to point how unaddressed variables can markedly affect the determination of whether a patient has an equinus condition, which should raise significant doubts in the reader that the 95\% confidence level is appropriate to consider rejecting the null hypothesis. Indeed, I feel that a more appropriate confidence level for this paper would be the $80 \%$, which would be more in line with the methods employed and also take into account some of the variables that I have elucidated. Again, this is not to criticize the authors for the fine work they have done, and I applaud the authors for pointing out the weaknesses in the study. However, I would encourage these authors and others for future work to try to better define the term equinus and to be more precise in applying it when researching the relationship between the function of the Achilles and the various maladies and dysfunctions of the foot.

Disclaimer: The views in this article are exclusively those of the author and do not in any way represent the official views of the U.S. Department of Veterans Affairs nor any agency or branch of the U.S. federal government.

\section{References}

1. Phillips, RD “Normal Biomechanics [Chapter 3]". In Principles and Practices of Podiatric Medicine. Levy LA, Hetherington VJ, Bakotic BW (Eds), 2 ${ }^{\text {nd }}$ edition. Brooklandville, MD, Data Trace Publishing Co., 2006) 